\title{
A Methodology for Tumor Detection in MRI using a New q-Gabor Function as a Convolutional Filter
}

\author{
Vinicius de A. Silva, Lucas P. Laheras, Everton C. Acchetta, Paulo S. Rodrigues \\ Computer Science Department, Centro Universitário FEI \\ São Bernardo do Campo, SP, Brasil \\ unifvsilva@fei.edu.br, lucaslaheras@hotmail.com, eve.023@hotmail.com, psergio@fei.edu.br
}

\begin{abstract}
Convolutional Neural Networks (CNN) can achieve excellent computer-assisted diagnosis with a good amount of data. However, there is still a growing demand for specific data and information for training Machine Learning models, either for classification or other tasks such as segmentation. Towards this, the Data Augmentation (DA) technique can handle the small medical imaging dataset problem by generating artificial training data. In this context, Generative Adversarial Networks (GANs) can synthesize realistic images to increase the number of images in a dataset. Therefore, to maximize the $\mathrm{DA}$ efficiency in a $\mathrm{CNN}$ based tumor classification task, we propose using non-extensive Gabor filters as a convolutional layer kernel initializer. Our proposal has been tested in the BraTS15 dataset and results show that CNN with an additional $q$-Gabor layer can achieve an average accuracy $3.65 \%$ better than $\mathrm{CNN}$ with Gabor and $5.03 \%$ better than the default model when trained with artificial images (data augmentation).
\end{abstract}

Index Terms-data augmentation, GANs, synthetic medical imaging, gabor function, tumor detection

\section{INTRODUCTION}

Recently, the development of both hardware and software has expanded in practically all areas of technology, impacting the advancement of society and the expansion of accessibility and availability of information.

One of the areas that has benefited the most is medicine, where the amount of digital information based on images, text and signals is nowadays much greater than the amount of physical information. The healthcare industry has successfully used modern machine learning methods at all clinical stages, including pre-clinical, diagnostic, operative, and posttreatment [1], [2]. One of the main challenges in the medical imaging field is the automatic diagnosis for tumor detection [3], which is the focus of this article.

On the other hand, Convolutional Neural Networks (CNNs) are the fundamental techniques used in this area, mainly for tumor classification, location, detection, and segmentation. However, for better performance of these techniques, a large amount of data is required. Currently, for the training of these networks, researchers usually carry out the classic data augmentation, which consists of applying geometric transformations to the original images [4]-[6]. However, it has been proven that more modern models of Deep Learning, such as Generative Adversarial Networks (GANs), can generate new artificial yet realistic samples and improve the results [7].

The combination of these two methods is explored by [8] to increase the performance of a CNN to classify liver injuries, the authors applied data augmentation by combining the classical data augmentation (geometric transformations) and generation of new samples using a GAN. The results showed a accuracy improvement from $78.6 \%$ to $85.7 \%$ in the classifier.

Using two-step DA-based GAN, the authors of [9] aimed to maximize the DA effect with GAN combinations. In the first step, Progressive Growing GAN, presented by [10], was used to generate realistic/diverse $256 \times 256$ MRI samples. The authors tested these experiments in a CNN-based tumor detection, which increased from $93 \%$ to $96 \%$ the classifier accuracy.

Artificial data generation has been very successful in several image processing applications, especially after the advancement of neural networks [11]. These networks are structured in different ways. One of the main strategies is the use of activation functions in convolutional processes, as is the famous case of CNN. These functions can also be used for spatial filtering in several dimensions, and their parameterization is usually one of the primary metrics. Some of the best-known works in this context are [12]-[15].

For many decades, the use of standard functions for modeling scientific-technological problems has been widespread. Among these functions, probably the most known is the exponential function, whose versions and simple parameterization facilitate the understanding and implementation. In addition to the vast statistical field where this function has been applied for centuries, it is in digital image processing (DIP) that it has gained recent popularity, probably due to its frequent use in filtering in both the spatial and frequency domains, a process known as mathematical convolution.

However, in the machine learning area, this function, along with other variants, has been used with enthusiasm, producing exceptional results that are gaining more and more popularity. In this area, more specifically in deep neural networks, the Gaussian function has been successfully used both in the convolution stage, as a convolutional kernel, and in the fully connected flat phase, as an activation function.

In turn, a Gabor function is a better choice for modeling various real problems, especially biological ones [16]. It is known, for example, that the neuronal communication signal in the brain can be better represented by a Gabor function instead of well-known functions such as relu, hyperbolic or sigmoid tangent . In the specific case of convolutional layers, 
the Gabor function is more appropriate when the analyzed scene's direction is essential for analyzing the data. This direction information is usually captured with the strategy of using several Gabor convolutional filters, each with a different direction.

On the other hand, with the emergence of Tsallis's nonextensive statistics [17], [18], many physical systems reinvented themselves and the enthusiasm for applications in various areas grew. The impact on image processing and computer vision was evident, [19]-[22]. The general idea of this statistic is the adaptability of functions to physical systems called non-extensive, through the estimation of the so-called non-extensivity parameter $q$. One of the best-known examples of this adaptability can be found in the work of [22], where the authors demonstrate the effectiveness of segmentation with the so-called $q$-Entropy.

Therefore, this work proposes a methodology based on data augmentation and CNN with $q$-Gabor 2D function, used as kernels initializer in convolutional layers, for tumor detection in magnetic resonance images.

\section{GABOR Functions And Their ApPliCATIONS}

\section{A. The 1D Gabor Function}

Traditional Gabor functions can be used as filters for onedimensional signals. Gabor function $G$ in the complex domain is defined by the product of a Gaussian envelope $w$ with a sinusoidal $s$ in the complex domain [16], as is shown in Equation 1 as the following.

$$
G(X)=k e^{\theta i} w(\alpha X) s(X)
$$

where:

$$
\begin{gathered}
w(X)=e^{-\pi X^{2}} \\
s(X)=e^{(2 \pi f X) i}
\end{gathered}
$$

and $\alpha, k, \theta$ and $f$ are the parameters of the function applied in the vector $X$. Then, you can see the Gabor filter as two parts: one real and one imaginary [16], as shown in Fig. 1.
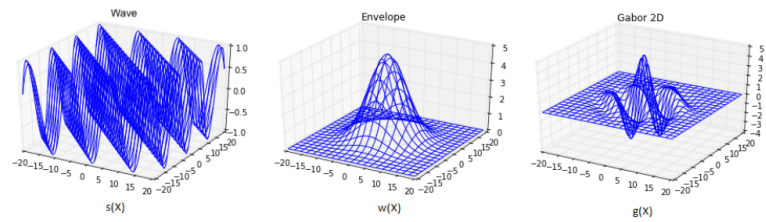

Fig. 1. Example of a 2D Gabor function with $u=v=0.08, P=0, k=5$, $X o=Y o=0, a=b=0.075$

\section{B. The 2D Gabor Function}

Similar to Equation 1 of the $1 D$ version, the $2 D$ version for the Gabor function's spatial domain is expressed in Equation 4.

$$
G(X, Y)=s(X, Y) w(X, Y)
$$

where $s(X, Y)$ is a sine wave in the complex domain called support, and $w(X, Y)$ is a 2D Gaussian form called envelope [16], best explained further.

The sinusoidal is defined by the function as the following complex domain:

$$
s(X, Y)=e^{(2 \pi(u X+v Y)+P) i}
$$

where $(u, v)$ and $P$ define spatial frequency and the sinusoidal frequency phase, respectively [16].

The envelope is defined as follows:

$$
w(X, Y)=k e^{-\pi\left(a^{2}(X-X o)^{2}+b^{2}(Y-Y o)^{2}\right)}
$$

where $(X o, Y o)$ is the envelope peak, and $a$ and $b$ are envelope width scaling parameters [16].

Finally, the complex Gabor Function consists of 9 parameters:

1) $(X, Y)$ : Location of the peak of the Gaussian envelope;

2) $u$ and $v$ : Spatial frequencies of the sinusoid carrier in Cartesian coordinates. It can also be expressed in polar coordinates as $\left(F_{0}, w_{0}\right)$;

3) $P:$ A phase of the sinusoid carrier;

4) $k$ : Scales the magnitude of the Gaussian envelope;

5) $a$ and $b$ : Scale the two axes of the Gaussian envelope;

6) $\theta$ : Rotation angle of the Gaussian envelope.

A Gabor function consists of two other functions, conveniently located in real and imaginary parts, forming a function in the complex domain [16]. An example of the 2D version is shown in Fig. 1.

\section{THE PROPOSAL FOR A $q$-GABOR FUNCTION}

It has been known for over a century that the entropy proposed by Boltzmann-Gibbs [23] has been able to explain several classical physical systems in the field of thermodynamics. However, for some systems with specific characteristics, such as long-range memory, long-range interactions, and fractal border behavior, Boltzmann's formalism is only an approximation. More detailed discussions of these ideas can already be found in the vast literature, as in [17], [18].

In the mid-1980s, C. Tsallis proposed a new formalism, which became known as Tsallis entropy or Tsallis statistics [17], [18]. This formalism was proposed in the form of Equation 7, whose main feature is the introduction of the socalled entropy parameter $q$, used to maximize the probability distritution $p_{i}, 0 \leq p_{i} \leq 1.0, \sum_{i=0}^{k} p_{i}=1.0$, where $k$ is the number of physical states of the system.

$$
S_{q}\left(p_{1}, \ldots p_{k}\right)=\frac{1-\sum_{i=1}^{k} p_{i}^{q}}{q-1}
$$

This parameter, when it tends to 1.0 , makes the (7) equivalent to the traditional entropy equation proposed by BoltzmannGibbs and Shannon (8), which became known in the area of Theory of Information as Shannon Entropy.

$$
S=-\sum_{i} p_{i} \ln \left(p_{i}\right)
$$


Regarding the vast number of successful applications of Tsallis statistics, specifically for DIP, and the recent and also good performances of the q-Gaussian and q-Sigmoid proposals [24], in this work, we present an extension of this theory applied to a 2D $q$-Gabor function used as a convolution kernel of a convolutional neural network for image classification. Like the proposal made for q-Sigmoid in [24], the proposal presented here for $q$-Gabor is replacing the Gaussian envelope with a q-Gaussian envelope. The details of this replacement are given further.

\section{A. The proposed q-Gabor 1D Function}

Similar to a Gabor function, a $q$-Gabor $q G$ is built with a sine wave $s$ and an envelope $w$ which is changed by adding a $q$-Exponential function, thus obtaining a thinner envelope next to its peak and broadest near base:

$$
\begin{gathered}
q G(X)=k e^{\theta i} w(\alpha X) s(X) \\
s(X)=e^{(2 \pi f X) i} \\
w(X)=\frac{1}{\left(1+(1-q) X^{2}\right)^{\frac{1}{1-q}}}
\end{gathered}
$$

Note that, in the envelope, the non-extensibility parameter $q$ cannot be equal to 1 due to the power denominator, which allowed us to guarantee to work in the non-extensive domain.

\section{B. The proposed q-Gabor 2D Function}

By expanding $q$-Gabor into the space domain, we get:

$$
\begin{gathered}
g(X, Y)=w(X, Y) s(X, Y) \\
s(X, Y)=e^{(2 \pi(u X+v Y)+P) i} \\
w(X, Y)=\frac{1}{(1+(1-q)(a(X-X o)+b(Y-Y o)))^{\frac{1}{1-q}}}
\end{gathered}
$$

Just as $q$-Gaussian functions tend to Gaussians when $q \rightarrow$ 1.0 , we hypothesize that $q$-Gabor functions try Gabor functions under the same conditions. This hypothesis has not been proven in the literature. However, the proof is not the focus of our work.

\section{The Proposed Methodology}

Traditionally, tumor detection in medical images is performed by adapting mathematical functions to probabilistic distributions. One of the most used functions is the so-called Gabor function. Considering that the non-extensive Tsallis entropy is an advance in this area when adapted to the $q$ Gaussian version, in this article, we propose a methodology using the $q$-Gabor function to solve this problem more broadly, since the $q$-Gabor is the generalization of the Gabor function just as the $q$-Gaussian is the generalization of the Gaussian function, with promising results.

This section presents the methodology proposed in this work. This methodology is composed of six steps, which the pipeline is presented in Fig. 2. This methodology is further explained in the following.

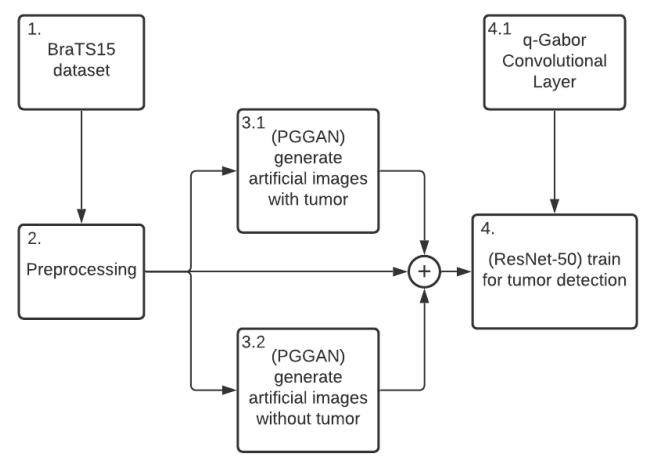

Fig. 2. Methodology flowchart.

\section{A. BraTS 2015}

The dataset used in this work consists of $240 \times 240$ contrastenhanced T1-weighted (T1c) brain axial MR images of highgrade glioma and low-grade glioma cases from Multimodal Brain Tumor Image Segmentation Benchmark (BRATS) 2015 [25].

\section{B. Pre-processing}

To perform the experiments, the dataset was extracted and separated as follows: slices were extracted from the range [60, 110] of 274 patients in total. During extraction, they were resized to $256 \times 256$ to better fit the PGGAN training . Finally, the slices were separated into tumor and non-tumor images. Thus, the final base was contained by:

- 7,592 tumor MRI samples of size $256 \times 256$.

- 1,064 non-tumor MRI samples of size $256 \times 256$.

Examples of samples from this step can be seen in Fig. 3.

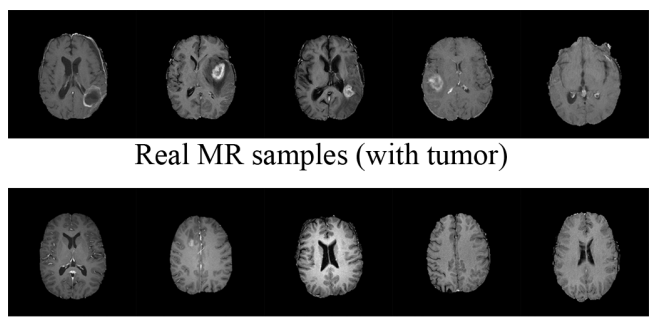

Real MR samples (without tumor)

Fig. 3. Examples of real MR samples of size $256 \times 256$.

1) Data split: After pre-processing step, the dataset was splitted into training, validation and test set for the classification task. Details of the sets can be observed in Table I.

TABLE I

REAL IMAGES SETS

\begin{tabular}{cccc}
\hline Set & Non-tumor & Tumor & Total \\
\hline Training set & 851 & 6.073 & 6.924 \\
Validation set & 106 & 759 & 865 \\
Test set & 107 & 760 & 867 \\
\hline
\end{tabular}




\section{Progressive Growing of GAN (PGGAN)}

The PGGAN architecture, denominated Progressive Growing of GAN [10], is a training methodology for GANs [26], which consists of progressively growing the generator and discriminator network. The training starts by generating lowresolution images, and new layers are added during this process until reaching the desired resolution. Accordingly to the authors, this speeds the training up and significantly stabilizes it, producing excellent artificial samples.

The training starts with the $G$ Generator Network and the $D$ Discriminator having a resolution of $4 \times 4$ pixels. As training process progresses, new layers are added for $G$ and $D$ so that the spatial resolution of the artificial images increases until the desired $256 \times 256$. Previous layers continue to be trained during this process [10].

1) PGGAN implementation details: This work uses PGGAN architecture to generate MRI samples with tumor and non-tumor artificially. Unlike the primary goal of generating high-resolution images $(1024 \times 1024)$ [10], we modified the network to generate $256 \times 256$ images.

\section{ResNet-50}

Residual Neural Networks are neural networks that consist of stacked residual units. Each unit can be expressed in the following:

$$
\begin{gathered}
y_{l}=h\left(x_{l}\right)+F\left(x_{l}, W_{l}\right) \\
x_{l+1}=f\left(y_{l}\right)
\end{gathered}
$$

where $x_{l}$ and $x_{l+1}$ are the input and output of the $l$-th unit, and $F$ is the residual function [27]. In [28], $h\left(x_{l}\right)=x_{l}$ is an identity mapping and $f$ is a ReLU function [29].

The primary idea of a Residual Neural Network is to learn the active residual function $F$ with respect to $h\left(x_{l}\right)$, with a key choice of using an identity mapping $h\left(x_{l}\right)=x_{l}$. This is accomplished by attaching an identity hopping, or shortcut connection [27].

1) ResNet-50 implementation details: This work used the residual model with 50 layers, ResNet-50. Some settings were modified to make it possible to detect tumors on MR images.

Similar to [9], a Dropout layer with a rate of 0.5 was introduced before the final Dense layer. The images were resized from $256 \times 256$ to $224 \times 224$ to fit the input size of ResNet and have been converted to grayscale (i.e., 1-channel). We use a bath size of 64 , and a learning rate of 0.002 with the SGD optimizer [30]. The loss function used was categorical crossentropy and the accuracy was used as a metric. This network will be mentioned as default in the sections below.

\section{E. q-Gabor 2D Convolutional Layer}

As shown in Section III-B, the $q$-Gabor 2D function was used as convolution filters (kernels), just as [31] used Gabor filters. The $q$-Gabor is a normal convolution layer; however, it is initialized with filters to fit the $q$-Gabor 2D function, and its values are automatically updated according to the network training. The layer is placed as the first convolution layer of the network [31] with a desired number of kernels and size, in this work we use 64 filters and a kernel size of $7 \times 7$. Table II shows the first layers of ResNet-50 architecture with the $q$-Gabor 2D as a convolutional layer.

TABLE II

INITIAL LAYERS OF RESNET50 ARCHITECTURE W/ $q$-GABOR

\begin{tabular}{cc}
\hline Layer & Output shape \\
\hline Input layer & $(224,224,1)$ \\
Zero padding & $(230,230,1)$ \\
$q$-Gabor2D $7 \times 7$ & $(\mathbf{1 1 2}, \mathbf{1 1 2}, \mathbf{6 4})$ \\
Conv2D $7 \times 7$ & $(53,53,64)$ \\
Max pooling & $(26,26,64)$ \\
\hline
\end{tabular}

\section{F. Experimental Configuration}

To compare the effects of data augmentation with images generated by PGGAN and also the use of $q$-Gabor 2D in tumor detection, the following ResNet50 configurations and image sets were compared:

1) Default ResNet-50

a) Training with real images;

b) Training with real $+88 \mathrm{k}$ PGGAN-based images;

2) ResNet-50 with Gabor convolutional layer

a) Training with real images;

b) Training with real + 88k PGGAN-based images;

3) ResNet-50 with $q$-Gabor convolutional layer

a) Training with real images;

b) Training with real $+88 \mathrm{k}$ PGGAN-based images;

To test these models, we added zero-mean white Gaussian Noise with the following variance values in the tests sets: 0.01 , $0.02,0.03,0.04,0.05,0.1,0.2,0,3,0.4,0.5$.

\section{RESUlTS AND Discussion}

In this work, PGGAN [10] was used for generating new realistic MR images. Fig. 4 show some examples of the realistic images generated by PGGAN in steps 3.1 and 3.2, respectively, of the proposed methodology (Fig. 2). It is possible to see

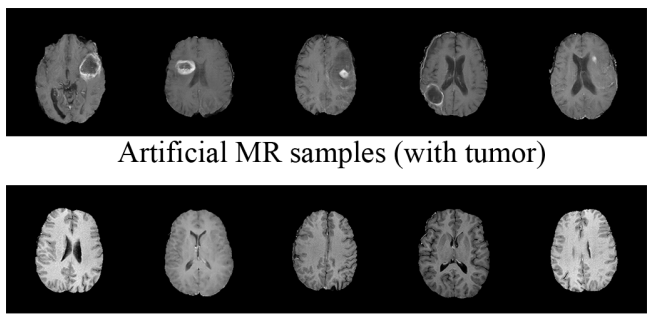

Artificial MR samples (without tumor)

Fig. 4. Examples of artificial MR samples of size $256 \times 256$.

that the artificially generated images are similar to the original images of the BraTS15 dataset. After performing steps 3.1 and 3.2, we generate $55.000 \mathrm{MR}$ images with tumor and 55.000 MR images without tumor.

For tumor detection, the PGGAN-generated images were separated into training, validation, and test images similar to 
TABLE III

ARTIFICIAL IMAGES SETS

\begin{tabular}{cccc}
\hline Set & Non-tumor & Tumor & Total \\
\hline Training set & 44.000 & 44.000 & 88.000 \\
Validation set & 5.500 & 5.500 & 11.000 \\
Test set & 5.500 & 5.500 & 11.000 \\
\hline
\end{tabular}

what was done in step 2. Details of the sets can be observed in Table III.

Table IV presents the results of the tumor detection task with the modifications made in ResNet-50. For ResNet-50 training, only the training, validation, and testing sets from the real dataset (Section IV-B1) were used. The images were resized from $256 \times 256$ to $224 \times 224$ to fit the input of the network. As a first experiment, the default architecture was used, and it was possible to achieve an accuracy of $99.30 \%$ by testing in 867 real images. As a second experiment, a convolutional layer with Gabor filters was added, as presented by [31], before the first convolutional layer. The model achieved an accuracy of $95.96 \%$. Like the previous experiment, the Gabor convolutional layer was replaced by a $q$-Gabor convolutional layer, and the model achieved an accuracy of $99.42 \%$.

TABLE IV

TUMOR DETECTION EXPERIMENTS

\begin{tabular}{cc}
\hline Training procedure & Accuracy \\
\hline ResNet-50 (no modifications) w/ real dataset & $99.30 \%$ \\
ResNet-50 (w/ Gabor2D) w/ real dataset & $95.96 \%$ \\
ResNet-50 (w/ qGabor2D) w/ real dataset & $\mathbf{9 9 . 4 2} \%$ \\
\hline
\end{tabular}

It can be noticed that the three network models performed well, but it is possible to notice the difference when the test set images are noisy. Fig. 5 presents the results obtained when adding Gaussian Noise with different values of variance to the images in the test set. It can be seen that the accuracy of the unmodified ResNet-50 (default) drops significantly as the images get noisier. On the other hand, ResNet with the Gabor layer and $q$-Gabor maintains accuracy up to $80 \%$ and $90 \%$, respectively. The model with $q$-Gabor maintains an accuracy up to $9.79 \%$ better than the model with Gabor. Table V

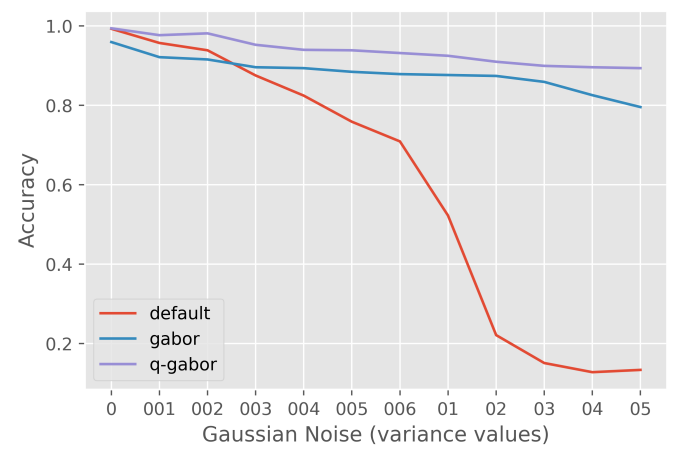

Fig. 5. Accuracy values evaluated in test set without data augmentation.

presents results when tests are carried out on the test set with artificial images, detailed in Table III. Accuracy drops from 3\% to $4 \%$ when the network is trained with a few set (real) images and tested on a large set of images (artificial). The architecture without additional convolution layers achieved an accuracy of $94.27 \%$, which is $3.17 \%$ better than the architecture using Gabor and $1.04 \%$ better than the architecture using $q$-Gabor.

TABLE V

Tumor Detection in DATA Aug. EXPERIMENTS

\begin{tabular}{cc}
\hline Training procedure & Accuracy \\
\hline ResNet-50 (no modifications) w/ real dataset & $\mathbf{9 4 . 2 7 \%}$ \\
ResNet-50 (w/ Gabor2D) w/ real dataset & $91.10 \%$ \\
ResNet-50 (w/ qGabor2D) w/ real dataset & $93.23 \%$ \\
\hline
\end{tabular}

However, when Gaussian Noise is added to the images, the model with the $q$-Gabor layer maintains a high accuracy up to 0.06 of variance, as shown in Fig. 6. On the other hand, the model with the Gabor layer does not have an accuracy very high from start but manages to maintain a good stability. The default model drops significantly to $48 \%$ of accuracy after a 0.03 of variance. Experiments 1-b, 2-b and 3-b, detailed

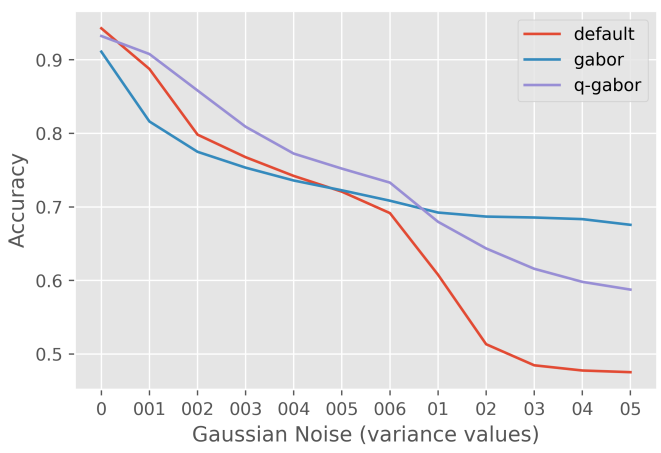

Fig. 6. Accuracy values evaluated in test set with $11 \mathrm{~K}$ images.

in Section IV-F were tested with the artificial images. The results are shown in Fig. 7. When tests are performed on images without any noise, the three models of the ResNet50 have good accuracy. However, as images become more noisy, the default model stays between gabor and $q$-gabor after a certain value of variance of Gaussian Noise. $q$-Gabor is superior compared to both gabor and default.

\section{CONCLUSION}

This work presented a methodology for detecting tumors in T1C MR images from the BraTS15 [25]. The proposed methodology used a PGGAN [10] for data augmentation, a ResNet-50 [28] for tumor detection and a $q$-Gabor $2 D$ function to initialize kernels for convolutional layer of the ResNet-50.

In order to increase images in the dataset, PGGAN was able to generate $110 \mathrm{~K}$ new realistic MRI images, separated into tumor images and non-tumor images. These images were used for training and testing the ResNet for tumor detection (binary classification). 


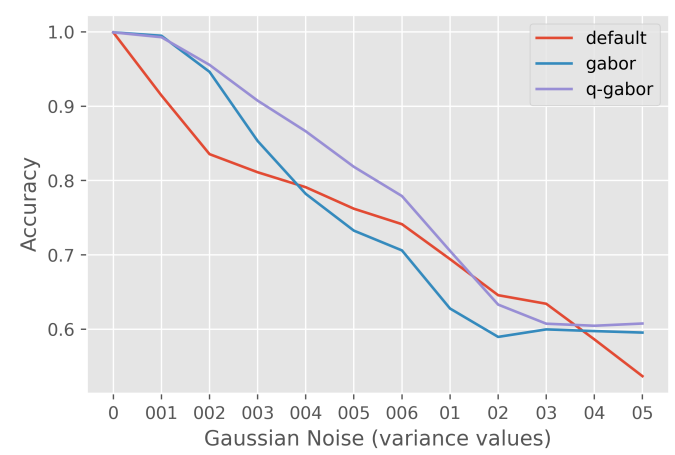

Fig. 7. Accuracy values evaluated in test set with model trained with data augmentation.w

To simulate the real world, such as when MR images can be collected with noise, we add Gaussian Noise to the test set images of both real and artificial images (generated by PGGAN).

For the experiments, three ResNet-50 models were used: (1) standard model, (2) model with a convolutional layer Gabor, (3) model with a convolutional layer $q$-Gabor. When training is done on a few images and tested on a large number of images (real + artificial), the standard models, with the Gabor and with the $q$-Gabor achieve an average accuracy of: $64.57 \%$, $73.71 \%$, and $74.08 \%$, respectively. When the artificial images combined with the real ones are used for training, the models reach an average accuracy of: $74.60 \%, 75.20 \%$, and $78.97 \%$, respectively.

Therefore, the results conclude that the model with the $q$ Gabor behaves better with and without data augmentation. Data augmentation increases the average accuracy of models by up to $7 \%$. These and other results with Tsallis entropy applied to medical imaging suggest the potential of this theory in future work using $q$-Gabor in CNNs.

\section{REFERENCES}

[1] A. S. Lundervold and A. Lundervold, "An overview of deep learning in medical imaging focusing on MRI," Zeitschrift fur medizinische Physik, vol. 29 2, pp. 102-127, 2019.

[2] J. Ker, L. Wang, J. P. Rao, and T. Lim, "Deep learning applications in medical image analysis," IEEE Access, vol. 6, pp. 9375-9389, 2018.

[3] P. K. Chahal, S. Pandey, and S. Goel, "A survey on brain tumor detection techniques for mr images," Multimedia Tools and Applications, pp. 144, 2020.

[4] F. Perez, C. N. Vasconcelos, S. Avila, and E. Valle, "Data augmentation for skin lesion analysis," in OR 2.0/CARE/CLIP/ISIC@MICCAI, 2018.

[5] C. N. Vasconcelos and B. N. Vasconcelos, "Experiments using deep learning for dermoscopy image analysis," Pattern Recognit. Lett., vol. 139, pp. 95-103, 2020.

[6] Y. Liu, S. Stojadinovic, B. A. Hrycushko, Z. Wardak, S. K. M. Lau, W. Lu, Y. Yan, S. B. Jiang, X. Zhen, R. Timmerman, L. A. Nedzi, and $\mathrm{X}$. Gu, "A deep convolutional neural network-based automatic delineation strategy for multiple brain metastases stereotactic radiosurgery," PLoS ONE, vol. 12, 2017.

[7] X. Yi, E. Walia, and P. S. Babyn, "Generative adversarial network in medical imaging: A review," Medical image analysis, vol. 58, p. 101552, 2019.
[8] M. Frid-Adar, I. Diamant, E. Klang, M. M. Amitai, J. Goldberger, and H. Greenspan, "Gan-based synthetic medical image augmentation for increased cnn performance in liver lesion classification," Neurocomputing, vol. 321, pp. 321-331, 2018.

[9] C. Han, L. Rundo, R. Araki, Y. Nagano, Y. Furukawa, G. Mauri, H. Nakayama, and H. Hayashi, "Combining noise-to-image and imageto-image gans: Brain MR image augmentation for tumor detection," IEEE Access, vol. 7, pp. 156966-156977, 2019.

[10] T. Karras, T. Aila, S. Laine, and J. Lehtinen, "Progressive growing of gans for improved quality, stability, and variation," ArXiv, vol. abs/1710.10196, 2018.

[11] C. Shorten and T. M. Khoshgoftaar, "A survey on image data augmentation for deep learning," Journal of Big Data, vol. 6, pp. 1-48, 2019.

[12] C. Nwankpa, W. L. Ijomah, A. Gachagan, and S. Marshall, "Activation functions: Comparison of trends in practice and research for deep learning," ArXiv, vol. abs/1811.03378, 2018.

[13] S. Sharma, S. Sharma, and A. Athaiya, "Activation functions in neural networks," 2020.

[14] L.-L. Huang, A. Shimizu, and H. Kobatake, "Classification-based face detection using gabor filter features," Sixth IEEE International Conference on Automatic Face and Gesture Recognition, 2004. Proceedings., pp. 397-402, 2004.

[15] G. Maguolo, L. Nanni, and S. Ghidoni, "Ensemble of convolutional neural networks trained with different activation functions," Expert Syst. Appl., vol. 166, p. 114048, 2021.

[16] J. R. Movellan. Tutorial on gabor filters. [Online]. Available: https://inc.ucsd.edu/mplab/tutorials/gabor.pdf

[17] C. Tsallis, "Nonextensive statistics: theoretical, experimental and computational evidences and connections," Brazilian Journal of Physics, vol. 29, pp. 1-35, 1999.

[18] — "Nonextensive statistical mechanics and its applications," 2001.

[19] G. A. Giraldi, P. S. S. Rodrigues, L. S. Marturelli, and R. L. S. Silva, "Improving the initialization, convergence, and memory utilization for deformable models," 2005.

[20] P. S. S. Rodrigues, R.-F. Chang, and J. S. Suri, "Non-extensive entropy for cad systems of breast cancer images," 2006 19th Brazilian Symposium on Computer Graphics and Image Processing, pp. 121-128, 2006.

[21] G. A. Giraldi, P. S. S. Rodrigues, R. L. S. Silva, A. L. ApolinárioJr., and J. S. Suri, "Level set formulation for dual snake models," 2007.

[22] P. S. S. Rodrigues and G. A. Giraldi, "Improving the non-extensive medical image segmentation based on tsallis entropy," Pattern Analysis and Applications, vol. 14, pp. 369-379, 2011.

[23] L. Boltzmann, S. G. Brush, and N. L. Balazs, "Lectures on gas theory," Physics Today, vol. 17, pp. 68-68, 1964.

[24] P. S. S. Rodrigues, G. A. Wachs-Lopes, R. M. Santos, E. Coltri, and G. A. Giraldi, "A q-extension of sigmoid functions and the application for enhancement of ultrasound images," Entropy, vol. 21, 2019.

[25] M. et al., "The multimodal brain tumor image segmentation benchmark (brats)," IEEE Transactions on Medical Imaging, p. 33, 2014. [Online]. Available: https://hal.inria.fr/hal-00935640

[26] I. J. Goodfellow, J. Pouget-Abadie, M. Mirza, B. Xu, D. Warde-Farley, S. Ozair, A. C. Courville, and Y. Bengio, "Generative adversarial networks," ArXiv, vol. abs/1406.2661, 2014.

[27] K. He, X. Zhang, S. Ren, and J. Sun, "Identity mappings in deep residual networks," ArXiv, vol. abs/1603.05027, 2016.

[28] — - "Deep residual learning for image recognition," 2016 IEEE Conference on Computer Vision and Pattern Recognition (CVPR), pp. 770-778, 2016.

[29] V. Nair and G. E. Hinton, "Rectified linear units improve restricted boltzmann machines," in ICML, 2010.

[30] L. Bottou, "Large-scale machine learning with stochastic gradient descent," in COMPSTAT, 2010.

[31] A. S. Alekseev and A. Bobe, "Gabornet: Gabor filters with learnable parameters in deep convolutional neural network," 2019 International Conference on Engineering and Telecommunication (EnT), pp. 1-4, 2019. 